\title{
Efficiency of voluntary opening hand and hook prosthetic devices: 24 years of development?
}

\author{
Gerwin Smit, MSc; ${ }^{*}$ Raoul M. Bongers, MSc, PhD; ${ }^{2}$ Corry K. Van der Sluis, MD, PhD; ${ }^{3}$ Dick H. Plettenburg, \\ MSc, PhD $^{1}$ \\ ${ }^{1}$ Delft Institute of Prosthetics and Orthotics, Department of Biomechanical Engineering, Delft University of Technol- \\ ogy, Delft, the Netherlands; ${ }^{2}$ Center of Human Movement Sciences and ${ }^{3}$ Department of Rehabilitation Medicine, Uni- \\ versity Medical Center Groningen, University of Groningen, Groningen, the Netherlands
}

\begin{abstract}
Quantitative data on the mechanical performance of upper-limb prostheses are very important in prostheses development and selection. The primary goal of this study was to objectively evaluate the mechanical performance of adultsize voluntary opening (VO) prosthetic terminal devices and select the best tested device. A second goal was to see whether VO devices have improved in the last two decades. Nine devices (four hooks and five hands) were quantitatively tested (Hosmer model 5XA hook, Hosmer Sierra 2 Load VO hook, RSL Steeper Carbon Gripper, Otto Bock model 10A60 hook, Becker Imperial hand, Hosmer Sierra VO hand, Hosmer Soft VO hand, RSL Steeper VO hand, Otto Bock VO hand). We measured the pinch forces, activation forces, cable displacements, mass, and opening span and calculated the work and hysteresis. We compared the results with data from 1987. Hooks required lower activation forces and delivered higher pinch forces than hands. The activation forces of several devices were very high. The pinch forces of all tested hands were too low. The Hosmer model 5XA hook with three bands was the best tested hook. The Hosmer Sierra VO hand was the best tested hand. We found no improvements in VO devices compared with the data from 1987.
\end{abstract}

Key words: body powered, efficiency, hysteresis, prosthetic design, prosthetic evaluation, prosthetic hand, prosthetics, qualitative testing, upper limb, voluntary opening.

\section{INTRODUCTION}

Despite the developments made in electrical prostheses, a significant number of adults and children wear bodypowered prostheses [1-2]. These users often prefer the relative benefits of the body-powered prostheses, such as low weight, technical reliability, low cost, and proprioceptive feedback, over the benefits of electrical prostheses, such as grip strength and the fact that a harness is not necessary in most cases. Body-powered prostheses, however, also have a number of drawbacks. A major complaint is activation force, which is often quite large [3]. This is uncomfortable and can lead to complaints and irritation of the shoulder and the axilla [4-5]. A further problem is frequent failure of the activation cable [4]. Although lighter than electrical devices, body-powered devices are also often perceived to be heavy by their users [4]. This article determines the mechanical efficiency of currently available body-powered voluntary opening (VO) hands and hooks. This helps patients select an appropriate device and manufacturers improve their designs.

\footnotetext{
Abbreviations: $\mathrm{VC}=$ voluntary closing, $\mathrm{VO}=$ voluntary opening. *Address all correspondence to Gerwin Smit, MSc; Delft Institute of Prosthetics and Orthotics, Department of Biomechanical Engineering, Delft University of Technology, Mekelweg 2, 2628 CD, Delft, the Netherlands; +31152781688. Email: g.smit@tudelft.nl
}

http://dx.doi.org/10.1682/JRRD.2011.07.0125 
To efficiently and smoothly use a body-powered prosthesis, the device must be mechanically efficient and require a low activation force. A previous study on voluntary closing (VC) devices showed that, except for the TRS hook, nearly all tested devices were inefficient and required high activation forces that users might find uncomfortable [6]. It is therefore interesting to study the performance of VO devices. The study described in this article objectively measures the mechanical performance of VO terminal devices for adults. On the basis of these measurements, it is possible to compare the performance and the efficiency of the tested devices.

No recent data are available on the mechanical efficiency of VO devices. Corin et al. published measurements on a broad range of adult- and child-size VO devices in 1987 [7]. Around that time, Carlson and Long also measured one VO and one VC hook [8], and LeBlanc et al. measured several VO and VC child-size prehensors [9]. This article presents new test data obtained from experiments similar to that of Corin et al. [7]. Comparing the current findings with those of Corin et al. makes it possible to give an objective view of how $\mathrm{VO}$ terminal devices have improved during the past decades. In the clinic, the results can be used to select an appropriate prosthetic terminal device for a patient. Manufacturers can also use the results to improve their prosthetic components.

The primary goal of this study was to objectively compare different VO terminal devices for adults by quantitatively measuring the mechanical work, energy dissipation, maximum cable force and excursion, pinch force, opening span, and device mass. We used this comparison to select the most suitable hand and hook prostheses based on the measured mechanical characteristics. The second goal was to see whether VO devices have improved over the last two decades. Therefore, we compared the results with those from a study in 1987 [7].

\section{METHODS}

\section{Test Materials}

In this study, we tested nine VO terminal devices: four hooks (Figure 1) and five hands (Figure 2). All devices were of comparable size (around size $73 / 4$ ), which corresponds to a small adult male hand or a large adult female hand; all devices were for left-side use. All devices were brand new and never before used.
All the hooks have adjustable spring force settings. The Hosmer Sierra VO hand (Hosmer; Campbell, California) has an adjustable thumb, which has two different opening positions. These settings can be easily adjusted by the user. We tested the devices for each individual setting. Some devices have a spring that is adjustable by the prosthetist or manufacturer. We tested all devices with the manufacturer's settings.

\section{Test Equipment}

We used a simple test bench to measure the force and displacement of the activation cable (Figure 3). The test bench was custom-built and consisted of standard components. A linear variable differential transformer (Positek Ltd; Cheltenham, United Kingdom) measured the displacements and a load cell (Zemic; Etten-Leur, the Netherlands) measured the cable activation force. We measured the pinch forces with a custom-built double leave strain gauge load cell. The voltage of each load cell was amplified with an amplifier (SCAIME; Annemasse, France). We recorded all data using a data acquisition system (National Instruments; Austin, Texas).

\section{Test Protocol}

For each device, we measured the mass and the maximum opening span. Subsequently, we subjected each device to three different tests:

1. Open and close test (full opening): We slowly pulled the cable $(\sim 2 \mathrm{~mm} / \mathrm{s})$ until the device was fully opened. Then, we released the cable at about the same speed until the device was closed again. During this test, we measured the cable force and displacement.

2. Open and close test $(50 \mathrm{~mm})$ : Similar to test 1 . In this test, we opened the device until the minimum opening between the fingers was $50 \mathrm{~mm}$.

3. Pinch force test: We placed the pinch force sensor between the fingertips of the device and measured the pinch force for opening widths of 10, 20, and $30 \mathrm{~mm}$.

We tested both the hands and the hooks according to the same protocol. The hands were tested with and without a cosmetic glove. During the gloved tests, each hand was gloved with the standard PVC (polyvinyl chloride) glove recommended by the manufacturer of that hand. To normalize the mass data, we shortened the long-sleeved RSL Steeper VO hand cosmetic glove to a length similar to that of the other cosmetic gloves (around $10 \mathrm{~cm}$ below the wrist plane). During the ungloved tests, the hands that had an inner glove were tested with the inner glove still 

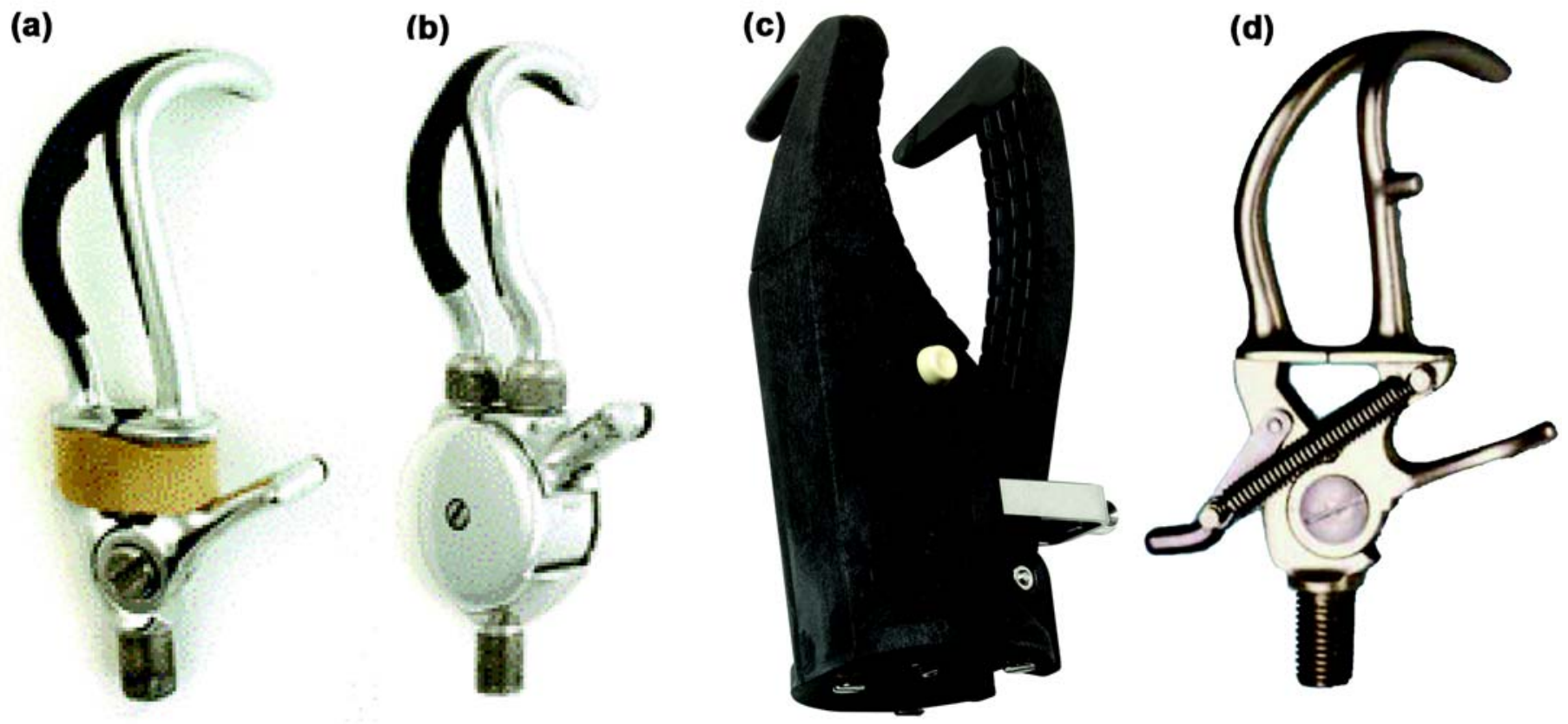

Figure 1.

Overview of tested hooks. Pictured devices are not actual measured devices. (a) Hosmer model 5XA hook (Hosmer; Campbell, California), (b) Hosmer Sierra 2 Load VO hook (Hosmer), (c) RSL Steeper Carbon Gripper (RSL Steeper; Leeds, United Kingdom), and (d) Otto Bock model 10A60 hook (Otto Bock; Duderstadt, Germany). VO = voluntary opening.

applied. We did not test the Becker Imperial hand (Becker Mechanical Hand Co; St. Paul, Minnesota) with a cosmetic glove because there was no matching glove available at the time of testing. Each test was preceded by two initial runs to prevent for transient behavior. We repeated tests 1 and 2 four times to obtain an average value and performed test 2 to enable a comparison among the different devices, since they all have different opening spans. Test 3 is a static test and therefore not repeated. The pinch force depends on the opening width of the fingers, not on their motion. The opening width before closing and pinching might have a small influence on the pinch force. To minimize this influence, we opened the devices only a few millimeters more than the thickness of the pinched object.

\section{Processing Data}

From the data, we determined the maximum excursion and activation force. To calculate the amount of energy (or work) needed to open the device, the measured forces were integrated along the displacement path. We also calculated the amount of energy that was "returned" during opening. The difference between the "input" and "output" energy is the dissipated energy, or hysteresis. This is an indicator of the efficiency of the mechanism. The higher the efficiency, the lower the hysteresis. Before processing the data, the start and end data with a cable force below $1 \mathrm{~N}$ were cut off because these data are not of interest.

\section{Comparison with 1987 Data}

We compared the acquired data with the data of the adult-size devices tested by Corin et al. in 1987 in a similar test [7]. This was done for the required work and activation force to fully open the device (test 1 ) and for the pinch force for small objects (test 3). We calculated the mean values of our data for the hooks and gloved hands in all settings. The mean values of Corin et al.'s data were calculated for the following adult-size hook devices: Hosmer model SSS-555 (1 to 3 bands), Hosmer model 88X (1 to 3 bands), and Hosmer model 99X (1 to 3 bands), and the gloved hand devices: UNB Steeper 2.50 in. and 2.75 in. (RSL Steeper; Leeds, United Kingdom); Hosmer Sierra VO hand; Hosmer Robins-Aids hand; Becker 
(a)

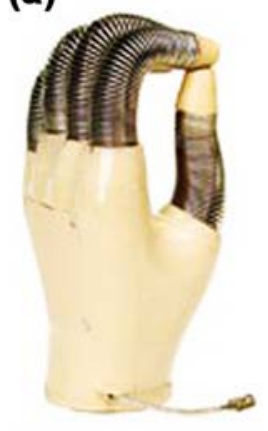

(b)

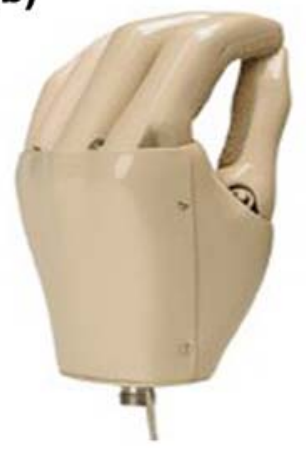

(c)

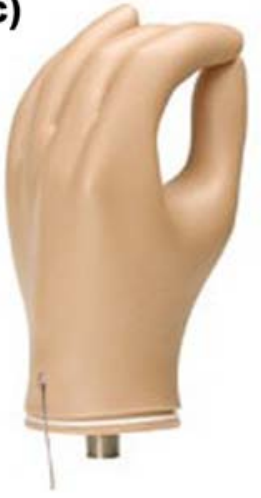

(d)

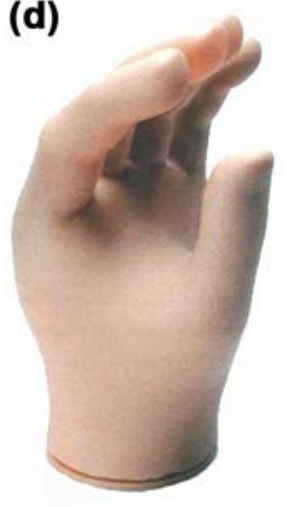

(e)

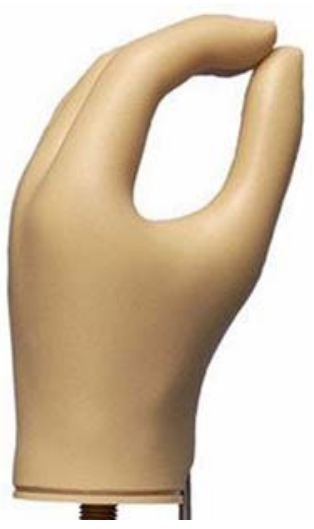

Figure 2.

Overview of tested hands without cosmetic glove applied. (a) Becker Imperial hand (Becker Mechanical Hand Co; St. Paul, Minnesota), (b) Hosmer Sierra VO hand (Hosmer; Campbell, California), (c) Hosmer Soft VO hand (Hosmer), (d) RSL Steeper VO hand (RSL Steeper; Leeds, United Kingdom), and (e) Otto Bock VO hand (Otto Bock; Duderstadt, Germany). Hands (a) and (b) do not have inner glove. Hands (c), (d), and (e) have inner glove, which protects cosmetic glove. $\mathrm{VO}=$ voluntary opening.

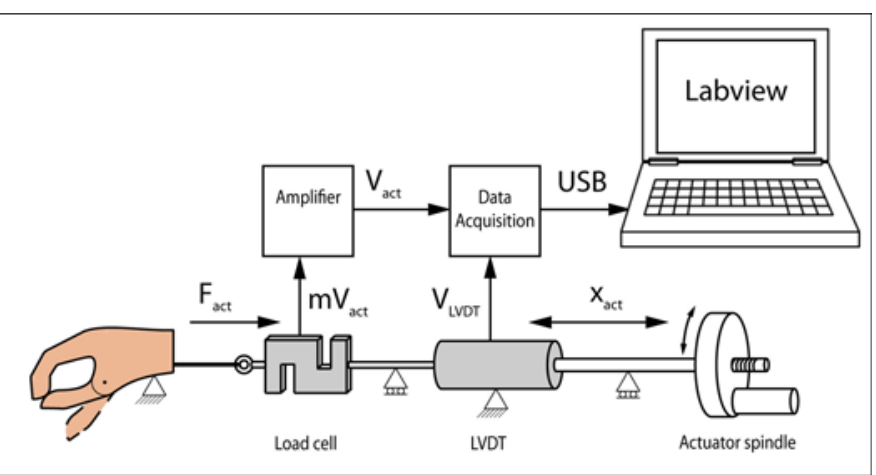

Figure 3.

Schematic overview of test setup during first two tests. Cable activation force and displacement are measured. $F_{\text {act }}=$ force activation cable, LVDT = linear variable differential transformer, $\mathrm{mV}_{\text {act }}=$ voltage force sensor (unamplified), USB = universal serial bus, $\mathrm{V}_{\mathrm{act}}=$ voltage force sensor (amplified), $\mathrm{V}_{\mathrm{LVDT}}=$ voltage displacement sensor, $\mathrm{X}_{\text {act }}=$ displacement activation cable.

Imperial hand; Hosmer models 201, 301, and 401; and Otto Bock 6.75 in. and 7.75 in. (Otto Bock; Duderstadt, Germany). We used an unpaired $t$-test to test whether significant $(\alpha<0.05)$ differences could be found between the data of both studies.

\section{Best Tested Prosthetic Devices}

Finally, it is interesting to see which hand and hook were most suitable for daily use according to the demands of the user. To enable a number of activities of daily living, the devices should have a pinch force above $20 \mathrm{~N}$ [10-11]. Their activation force should be as low as possible. The cable excursion should be within the acceptable range $(<53 \mathrm{~mm})$ [12]. The mass should be as low as possible. The results will be compared with these user demands.

\section{RESULTS}

The Table presents characteristics of the prosthetic devices, as well as an overview of all test data.

\section{Mass}

The measured hands had a mass 1.6 to 5.1 times higher than that of the hooks. The device with the lowest mass was the Hosmer model 5XA hook with one rubber band (87 g). The gloved Hosmer Sierra VO hand had the highest mass (447 g). The Becker Imperial hand was the heaviest ungloved hand (367 g).

\section{Maximum Opening Span}

The opening span of the hooks had a range from 67 (Otto Bock model 10A60 hook) to $97 \mathrm{~mm}$ (RSL Steeper Carbon Gripper). The span of the gloved hands ranged from 62 (Hosmer Sierra VO hand) to $80 \mathrm{~mm}$ (Otto Bock VO hand). Using a cosmetic glove reduced the opening span of the hands a few millimeters. 
Table.

Overview of test results of voluntary opening (VO) hand and hook tests.

\begin{tabular}{|c|c|c|c|c|c|c|c|c|c|c|c|c|c|}
\hline \multirow{2}{*}{$\begin{array}{l}\text { VO Terminal } \\
\text { Device }\end{array}$} & \multirow{2}{*}{$\begin{array}{l}\text { Mass } \\
\text { (g) }\end{array}$} & \multirow{2}{*}{$\begin{array}{l}\text { Max. } \\
\text { Open } \\
(\mathbf{m m})\end{array}$} & \multicolumn{2}{|c|}{$\begin{array}{c}\text { Max. Cable } \\
\text { Excursion } \\
(\text { mean } \pm \text { SD, } n=4) \\
\end{array}$} & \multicolumn{2}{|c|}{$\begin{array}{c}\text { Max. Cable Force } \\
(\text { mean } \pm \text { SD, } n=4)\end{array}$} & \multicolumn{2}{|c|}{$\begin{array}{c}\text { Open and Close } \\
\text { Test 1: Full } \\
(\text { mean } \pm \text { SD, } n=4)\end{array}$} & \multicolumn{2}{|c|}{$\begin{array}{c}\text { Open and Close } \\
\text { Test 2: } 50 \mathrm{~mm} \\
(\text { mean } \pm \text { SD, } n=4)\end{array}$} & \multicolumn{3}{|c|}{ Pinch Force Test 3} \\
\hline & & & $\begin{array}{c}\text { Test 1: } \\
\text { Full (mm) }\end{array}$ & $\begin{array}{c}\text { Test 2: } \\
50 \mathrm{~mm} \\
(\mathrm{~mm})\end{array}$ & $\begin{array}{c}\text { Test 1: } \\
\text { Full } \\
\text { (N) }\end{array}$ & $\begin{array}{l}\text { Test 2: } \\
50 \mathrm{~mm} \\
\text { (N) }\end{array}$ & $\begin{array}{l}\text { Work } \\
(\mathrm{Nmm})\end{array}$ & $\begin{array}{l}\text { Hysteresis } \\
\text { (Nmm) }\end{array}$ & $\begin{array}{l}\text { Work } \\
\text { (Nmm) }\end{array}$ & $\begin{array}{l}\text { Hysteresis } \\
\text { (Nmm) }\end{array}$ & $\begin{array}{c}10 \mathrm{~mm} \\
(\mathrm{~N})\end{array}$ & $\begin{array}{c}20 \mathrm{~mm} \\
(\mathrm{~N})\end{array}$ & $\begin{array}{l}30 \mathrm{~mm} \\
(\mathrm{~N})\end{array}$ \\
\hline \multicolumn{14}{|c|}{ Hosmer Model 5XA Hook* } \\
\hline 1 band & 87 & 88 & $45 \pm 0.2$ & $24 \pm 0.1$ & $48 \pm 12.0$ & $25 \pm 0.3$ & $1,128 \pm 14$ & $290 \pm 3$ & $574 \pm 3$ & $120 \pm 4$ & 9 & 9 & 9 \\
\hline 2 bands & 90 & 88 & $46 \pm 0.1$ & $25 \pm 0.1$ & $72 \pm 3.5$ & $50 \pm 0.2$ & $2,248 \pm 10$ & $394 \pm 6$ & $1,173 \pm 6$ & $154 \pm 3$ & 14 & 19 & 20 \\
\hline \multicolumn{14}{|c|}{ Hosmer Sierra 2 Load VO Hook ${ }^{*}$} \\
\hline Setting 1 & 242 & 66 & $34 \pm 0.1$ & $26 \pm 0.0$ & $67 \pm 7.9$ & $40 \pm 0.3$ & $1,243 \pm 11$ & $379 \pm 1$ & $868 \pm 1$ & $245 \pm 3$ & 9 & 11 & 11 \\
\hline Setting 2 & 242 & 66 & $35 \pm 0.0$ & $26 \pm 0.0$ & $117 \pm 6.4$ & $82 \pm 0.1$ & $2,642 \pm 14$ & $571 \pm 2$ & $1,820 \pm 2$ & $337 \pm 2$ & 24 & 27 & 29 \\
\hline \multicolumn{14}{|c|}{ RSL Steeper Carbon Gripper ${ }^{\dagger}$} \\
\hline Setting 1 & 171 & 97 & $43 \pm 0.2$ & $28 \pm 0.1$ & $70 \pm 0.4$ & $43 \pm 0.3$ & $1,619 \pm 2$ & $487 \pm 4$ & $846 \pm 4$ & $267 \pm 4$ & 11 & 11 & 11 \\
\hline $\begin{array}{l}\text { Hosmer Becker } \\
\text { Imperial Hand } \\
\text { (ungloved) }^{\Uparrow}\end{array}$ & 367 & 75 & $49 \pm 0.3$ & $30 \pm 0.0$ & $65 \pm 0.3$ & $63 \pm 0.4$ & $2,748 \pm 17$ & $1,710 \pm 9$ & $1,613 \pm 9$ & $1,031 \pm 6$ & 10 & 11 & 10 \\
\hline \multicolumn{14}{|c|}{ Hosmer Sierra VO Hand ${ }^{*}$} \\
\hline Ungloved & 356 & $41 / 67^{* *}$ & $25 \pm 0.1$ & $16 \pm 0.1$ & $75 \pm 1.7$ & $52 \pm 0.7$ & $1,152 \pm 8$ & $637 \pm 6$ & $581 \pm 6$ & $313 \pm 1$ & 15 & 12 & 10 \\
\hline Gloved & 447 & $38 / 62^{* *}$ & $22 \pm 0.3$ & $16 \pm 0.3$ & $84 \pm 0.8$ & $70 \pm 0.6$ & $1,017 \pm 24$ & $583 \pm 11$ & $639 \pm 11$ & $358 \pm 9$ & 7 & 11 & 18 \\
\hline \multicolumn{14}{|c|}{ Hosmer Soft VO Hand* } \\
\hline Ungloved & 310 & 74 & $38 \pm 0.1$ & $30 \pm 0.2$ & $121 \pm 0.9$ & $81 \pm 0.9$ & $2,170 \pm 16$ & $858 \pm 6$ & $1,406 \pm 6$ & $536 \pm 10$ & 15 & 12 & 12 \\
\hline Gloved & 400 & 70 & $36 \pm 0.3$ & $32 \pm 0.1$ & $138 \pm 4.5$ & $104 \pm 0.9$ & $2,266 \pm 29$ & $1,082 \pm 9$ & $1,690 \pm 9$ & $806 \pm 19$ & 12 & 12 & 14 \\
\hline \multicolumn{14}{|c|}{ RSL Steeper VO Hand ${ }^{\dagger}$} \\
\hline
\end{tabular}

\section{Maximum Cable Excursion}

The maximum cable excursion ranged from 34 (Hosmer Sierra 2 Load VO hook) to $46 \mathrm{~mm}$ (Hosmer model 5XA hook) for the hooks and from 22 (Hosmer Sierra VO hand) to $53 \mathrm{~mm}$ (Otto Bock VO hand) for the gloved hands.

\section{Activation Force}

Figure 4 shows the maximum required activation forces during the $50 \mathrm{~mm}$ open and close test (test 2).
Applying a cosmetic glove increased the activation force by 22 to 41 percent in this test.

Figures $\mathbf{5}$ and $\mathbf{6}$ depict the force displacement graphs of the full open and close test (test 1). The graphs show the different slopes of the devices caused by the differences in stiffness. The graphs also show that the return trajectory is lower than the opening trajectory. This is caused by the mechanical friction in the mechanism and by the internal friction in cosmetic glove and in the stiff 
inner glove. The Otto Bock model 10A60 hook (setting 1 ) is the only device that has a decreasing activation force characteristic (Figure 5). Another remarkable phenomenon can be seen in the plot of the Hosmer Soft VO hand (Figure 6). The force displacement graph shows a sawtooth pattern.

\section{Work and Hysteresis}

The bar charts (Figure 7) enable easy comparison of the work and hysteresis measured for the devices in the $50 \mathrm{~mm}$ open and close test (test 2). In this test, the hooks dissipated 11 (Hosmer model 5XA hook, 3 bands) to 46 percent (Otto Bock model 10A60 hook, setting 1) of the input energy. The ungloved hands dissipated 35 (Otto Bock VO hand) to 64 percent (Becker Imperial hand), and the gloved hands dissipated 43 (Otto Bock VO hand) to 56 percent (Hosmer Sierra VO hand) of their input energy.

\section{Pinch Forces}

The measured pinch forces of the hooks ranged from 9 (Hosmer model 5XA hook, 1 band) to $37 \mathrm{~N}$ (Otto Bock model 10A60 hook, setting 2). The pinch forces of the gloved hands ranged from 7 (Hosmer Sierra VO hand: $10 \mathrm{~mm}$ object; RSL Steeper VO hand: $20 \mathrm{~mm}$ object) to 18 N (Hosmer Sierra VO hand: 30 mm object).

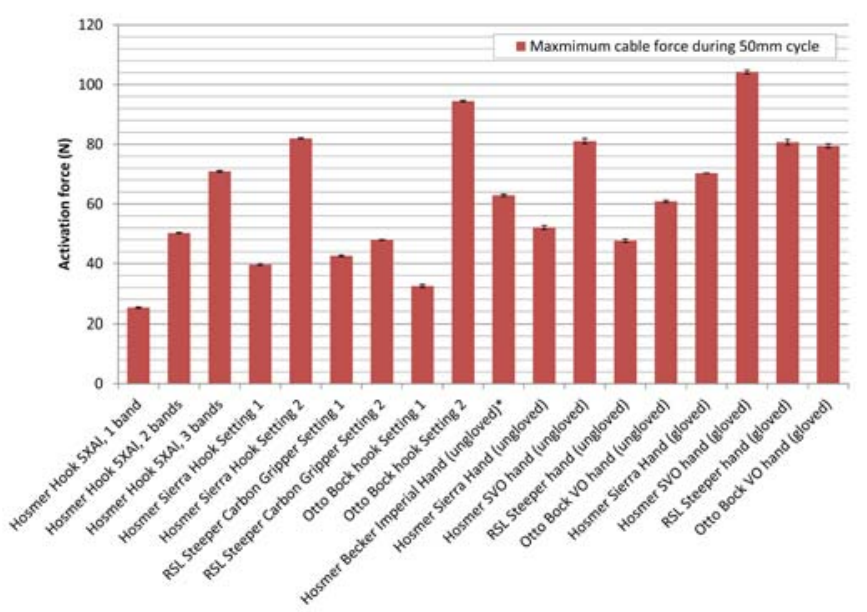

\section{Figure 4.}

Maximum cable activation force to open devices $50 \mathrm{~mm}$ during test 2. ${ }^{*}$ Becker Imperial hand (Becker Mechanical Hand Co; St. Paul, Minnesota) was only tested without cosmetic glove. SVO = soft voluntary opening, $\mathrm{VO}=$ voluntary opening.

\section{Best Tested Prosthetic Devices}

Of the hooks that could pinch over $20 \mathrm{~N}$, the Hosmer model 5XA hook with 3 bands (24-33 N) required the lowest activation force $(95 \pm 4 \mathrm{~N})$. Its cable excursion (46 \pm $0 \mathrm{~mm}$ ) was within the acceptable range $(<53 \mathrm{~mm})$, and it had the lowest mass (92 g).

None of the hands complied with the demand of having a pinch force above $20 \mathrm{~N}$. The hands that had the largest pinch force with the cosmetic glove applied were the Hosmer Sierra VO hand (7-18 N) and the Hosmer Soft VO hand (12-14 N). Of all hands, the Hosmer Sierra VO hand required the lowest activation force $(84 \pm 0.8 \mathrm{~N})$.

\section{Comparison with 1987 Data}

Figure 8 shows the mean \pm standard deviation values of the devices tested in this study and of the adult devices tested by Corin et al. [7]. The $p$-values of the $t$-test are presented below each plot. There is only a significant difference for the activation force of the hooks and hands. All other parameters did not differ significantly $(p>0.05)$.

\section{DISCUSSION}

The goal of this study was to objectively compare current VO prosthetic terminal devices among each other by using quantitative measurements. We used the results to select the best tested hand and hook and to see whether VO devices have improved over the last decades.

\section{Mass}

The human hand has a mass of $400 \pm 90 \mathrm{~g}$ [13]. The mass of a prosthetic device should be considerably lower to enable comfortable wearing. All measured hooks were significantly lighter (87-242 g) than a human hand. The gloved hands were of the same weight as the human hand (395-447 g). This might be one reason that a high weight is also an issue in body-powered devices [4] and why hooks are preferred over hands. The results showed that the cosmetic glove significantly contributes to the mass of the hand. The Becker Imperial hand was the heaviest hand without a cosmetic glove, and it may also have the highest mass with cosmetic glove applied.

\section{Cable Excursion}

For a harness using "arm-flexion control," Taylor measured a maximum cable excursion of $53 \pm 10 \mathrm{~mm}$ [12]. All hooks and gloved hands were within this range. The Hosmer model 5XA hook, the gloved Otto Bock and 

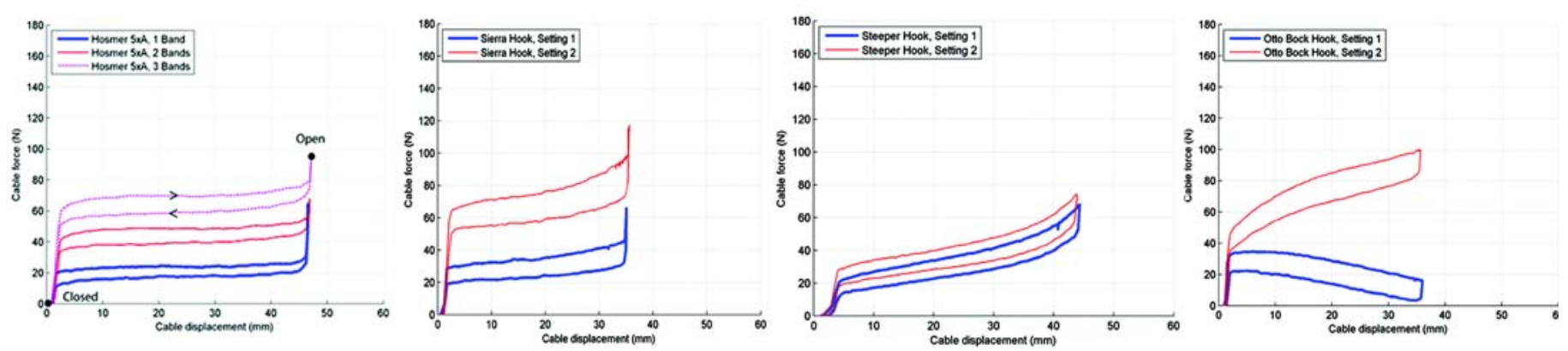

Figure 5.

Force displacement graphs of hook devices in their different settings during full open and close test.

RSL Steeper VO hands, and the ungloved RSL Steeper VO and Becker Imperial hands were not within the range minus one standard deviation (43 mm). Only the Hosmer Sierra VO hand was within the range minus two times the standard deviation $(33 \mathrm{~mm})$. This implies that not all users can fully open all devices.

\section{Level of Activation Force}

The maximum activation forces of all devices were well within the maximum activation force of shoulder control (280 $\pm 24 \mathrm{~N})$ as measured by Taylor [12]. However, his data were measured on nondisabled subjects. Shaperman et al. show that children with a congenital upper-limb deficiency had lower strength in both their deficient and their sound arm than nondisabled children ( 1.5-2.0 times lower) [14]. Furthermore, Taylor only measured maximum forces [12]. However, the critical force is not known. The critical force is the percentage of the maximum force that a person can exert over a certain period of time without getting fatigued [15]. Because VO devices are usually only opened for short periods of time, it is difficult to determine the critical force. From the literature, it is not clear whether the required forces to operate a prosthetic hand during daily life activities are a problem for the user. Therefore, further research on this topic is needed.

The decreasing force characteristic (Figure 5) of the Otto Bock model 10A60 hook (setting 1) is caused by the configuration of the spring in this setting. During opening, the distance between the spring and the joint decreases to close to zero. As a result, the hook almost acts like a bistable mechanism, which is stable when it is closed or fully open but unstable in positions in between. Although this makes it easier to keep the hook fully open, it may be more difficult to accurately control the opening width of the hook in between these extreme positions. The jerky behavior of the Hosmer Soft VO hand (Figure 6) is probably caused by stick-slip behavior of the mechanism. This behavior could make it difficult to accurately control the hand.

\section{Level of Pinch Force}

A pinch force of $10 \mathrm{~N}$ is considered to be sufficient for most activities of children [16]. For adults, the desired pinch is about two times higher and occasionally more. Van Der Niet et al. show that an iLimb, which had a maximum pinch force of 15 to $20 \mathrm{~N}$, did not exert enough force to complete all tasks [11]. Keller et al. show that the required pinch force is even higher for several activities (e.g., holding a tea cup: $28 \mathrm{~N}$; pulling on a sock: $34 \mathrm{~N}$ ) [10]. Therefore, it is assumed that the VO devices should be able to pinch over $20 \mathrm{~N}$. In their highest setting, all hooks could pinch well over $20 \mathrm{~N}$ except for the RSL Steeper Carbon Gripper, which had a maximum pinch force of $14 \mathrm{~N}$. None of the tested hands could pinch over $20 \mathrm{~N}$. The maximum measured pinch force was $18 \mathrm{~N}$ in the gloved Hosmer Sierra VO hand. All other measured hand pinch forces did not exceed $15 \mathrm{~N}$. These results indicate that the produced pinch forces of the VO hands are not large enough to complete all tasks of daily living. The low pinch forces measured for the hands and the relative high pinch forces measured for the hooks might be an important reason for the high rejection rates of body-powered hands and the relative good acceptance for hooks.

Note that the method of the pinch force test we employed differed from that of Corin et al. [7]. They attached the fingers of the fully opened hand to the test bench using two vertical spanned cables. The pinch force was then measured while the cables were slowly released. In this method, the hand is only supported by the cables. Initial trials in our study showed that the measured pinch 

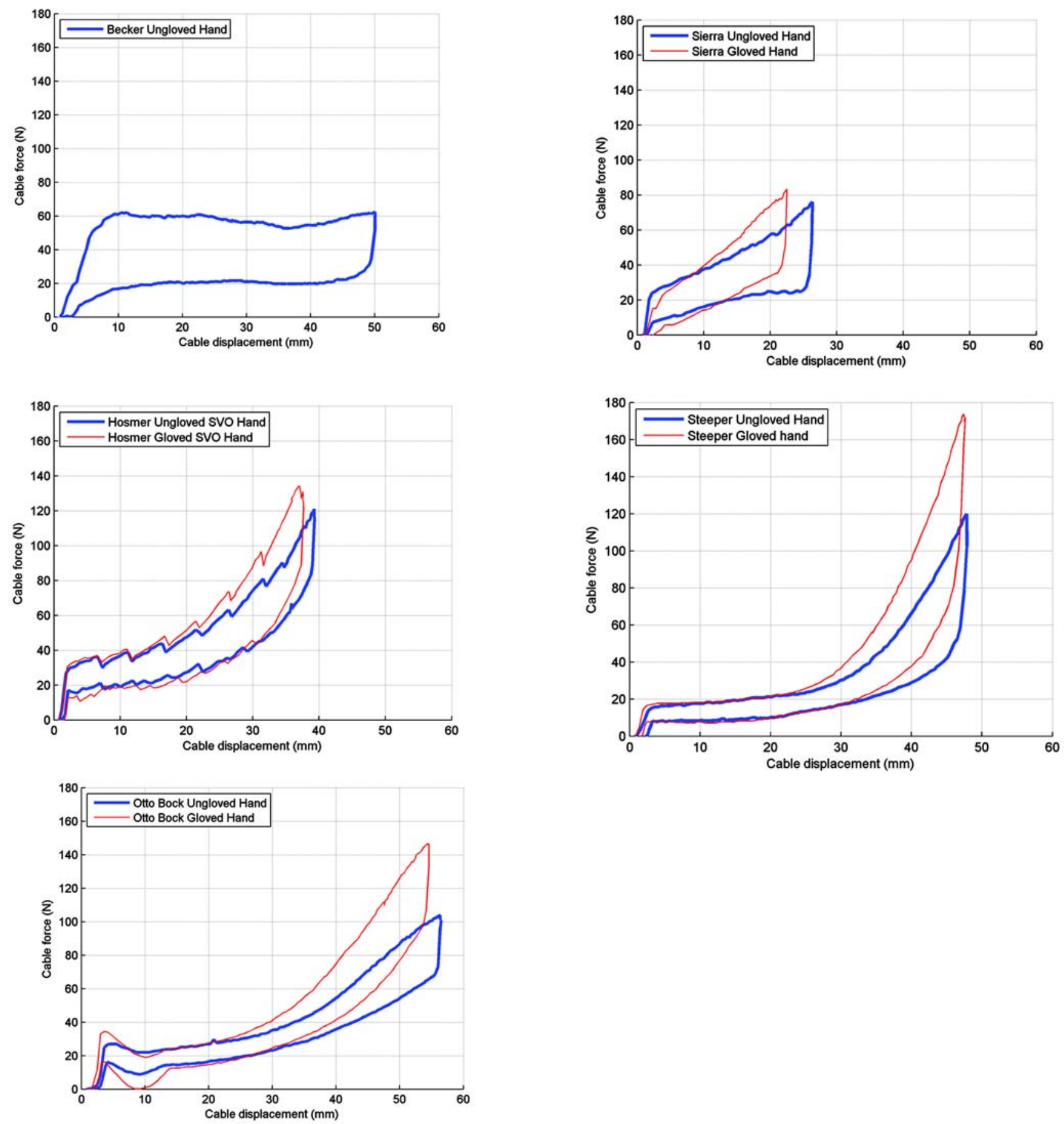

Figure 6.

Force displacement graphs of hand devices, with and without cosmetic glove applied, during full open and close test. Becker Imperial hand (Becker Mechanical Hand Co; St. Paul, Minnesota) was only tested without glove. SVO = soft voluntary opening.

force was influenced by the mass of the device, which was not in line with the vertical cables. The outcomes were also influenced when the cables were spanned horizon- tally, especially for small finger openings. When Corin et al.'s method is used, the measured pinch forces can be higher than the actual pinch force [7]. This effect is larger 


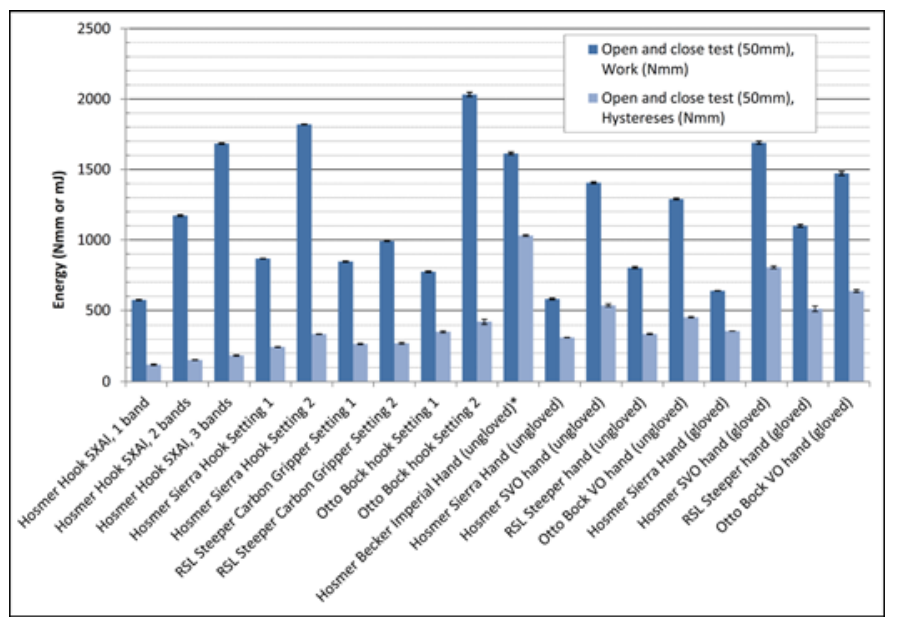

Figure 7.

Required work to open devices $50 \mathrm{~mm}$ and energy dissipated during one cycle of opening and closing $50 \mathrm{~mm}$ during test 2 . *Becker Imperial hand (Becker Mechanical Hand Co; St. Paul, Minnesota) was only tested without cosmetic glove. SVO = soft voluntary opening, $\mathrm{VO}=$ voluntary opening.

for hands than for hooks because of their larger mass. Although these deviations are relatively small, it is more accurate to measure the pinch force directly. Therefore, in our study we used a pinch force sensor to measure the pinch force for three opening spans. Because the overestimation of the pinch force in the data of Corin et al. would be small, and because the standard deviations are large (Figure 8(b)), it is unlikely that this has affected the outcome of the pinch force comparison significantly [7].

\section{Best Performing Devices}

It was easy to select the best-performing prosthetic hook. In general, the hooks performed much better than the hands. Selecting a suitable hand was difficult because none of the hands produced a sufficient pinch force. Using the hands for activities of daily living is therefore expected to be limited.

\section{Past, Present, Future}

It is interesting to compare the results with the data of the test performed by Corin et al. in 1987 [7]. In the past two decades, many new materials have become available that can be used for mechanism, bearings, gloves, etc. Meanwhile, various user studies clearly mapped the needs of the prosthesis user [4,17]. However, comparisons between our results and Corin et al.'s results showed no significant difference for the required work or pinch forces for the hooks or for the hands (Figure 8(a)
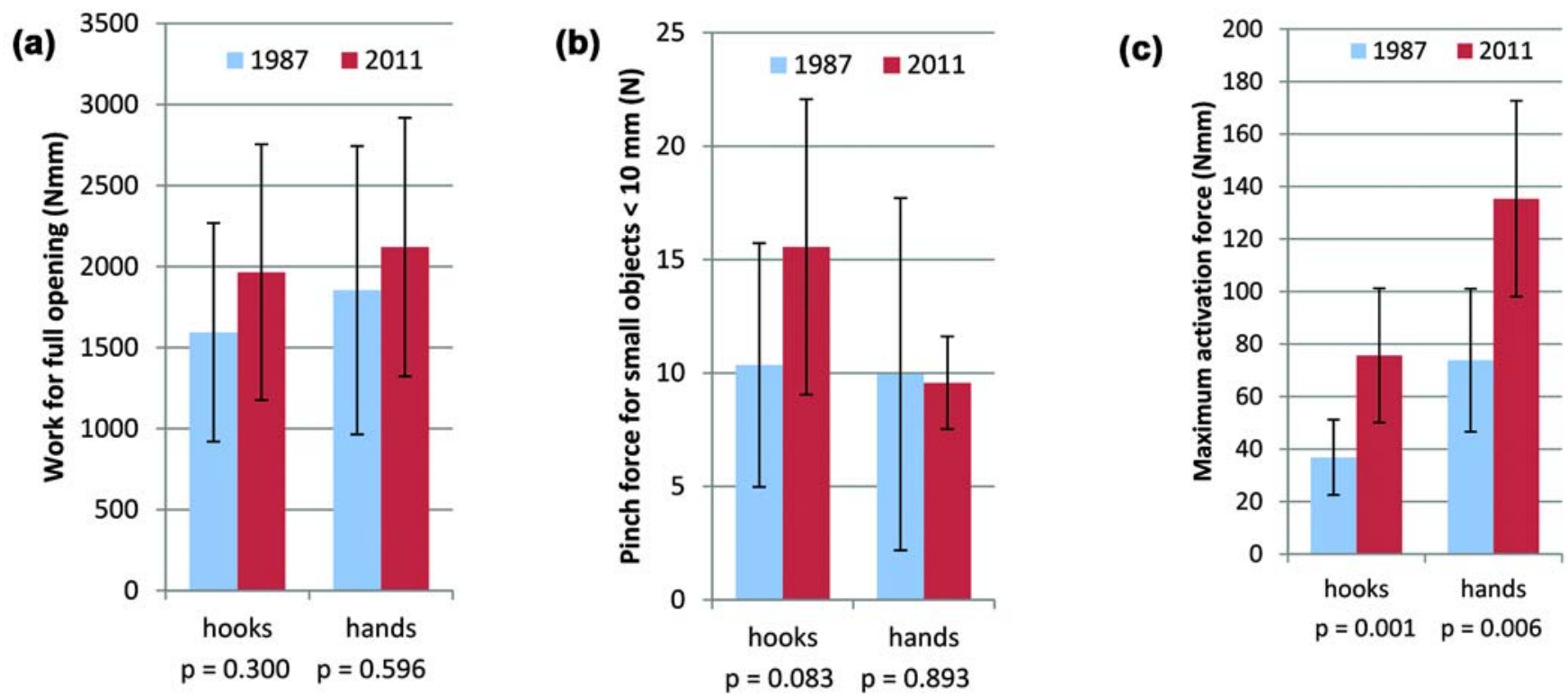

Figure 8.

Comparison between (a) values of work, (b) pinch force for small objects, and (c) activation force of hooks and hands tested in 1987 and in 2011. 
and 8(b)). However, the activation force for the hands and the hooks was significantly higher in this study (Figure 8(c)), which means that they performed worse. In general it can therefore be concluded that, despite all technologic advantages in other fields, VO prosthetic devices have not improved since 1987.

This study also shows that some newer devices had a poorer performance than devices tested in 1987. For example, the RSL Steeper Carbon Gripper required a maximum activation force of $75.0 \pm 0.2 \mathrm{~N}$, and it delivered a pinch force of $14 \mathrm{~N}$. The Hosmer model SSS-555 hook and the Hosmer model 10P hook, tested by Corin et al. in 1987, required a lower activation force of 49 and $65 \mathrm{~N}$, respectively [7]. They also delivered a higher pinch force of 15 and $26 \mathrm{~N}$, respectively. Another example is the current Otto Bock VO hand, which required an activation force of $146.0 \pm 1.7 \mathrm{~N}$ and delivered a pinch force of $9 \mathrm{~N}$ for objects of $10 \mathrm{~mm}$. Its predecessor, the Otto Bock VO hand tested in 1987, required an activation force of $62 \mathrm{~N}$ and delivered a pinch force of $14 \mathrm{~N}$ for small objects.

The outcome of this comparison with the data from 1987 raises a number of questions:

- Why have VO devices not been improved over the past decades? This is especially interesting to know for hands, which still have an insufficient pinch force and a high activation force that may be uncomfortable.

- How is it possible that new devices have become available that perform worse than their predecessors? Why do prosthesis users not benefit from apparent innovations?

Perhaps the most interesting question is-

- What would be the real future potential of bodypowered prostheses if we would invest the same effort and resources into the improvement of body-powered prostheses that is currently invested in electric devices?

For now, these questions remain unanswered; however, they deserve studying. There are many opportunities for improvements in body-powered prosthetics, especially for prosthetic hands:

- Mass of the hands should be reduced to enable comfortable wearing.

- Activation force should be reduced to a comfortable level. A study should reveal the comfortable force level. Moreover, the optimal shoulder movement to produce the most efficient force should be examined.
- Pinch force should be increased to an acceptable level $(>30 \mathrm{~N})$. To enable this, the mechanism should be more efficient. This could be achieved by redesigning the mechanism and by improving the bearings of the mechanism.

- More flexible cosmetic gloves should be developed.

- Inner glove should be more flexible, or its use should be avoided.

Indications in literature show that overload of the contralateral shoulder might lead to symptoms of overuse [18]. Therefore, improvement of body-powered prostheses is not only desirable but a necessity. Prosthesis users should be offered a prosthesis that is optimized to their needs and demands.

\section{Study Limitations}

Of each prosthetic device, we only tested one specimen. Also, for each cosmetic glove type, we only tested one glove. Variations in individual gloves, prostheses, and factory spring settings might result in deviations from the measured data. These effects are expected to be the smallest in the hooks and the largest in the hands with adjustable spring settings and a cosmetic glove. From cosmetic gloves, we know that their properties can vary because of variations in thickness [19]. Although some variables might cause variations in the results for the gloved hands, they are expected to give only minor variations for the hooks and the ungloved hands. Testing multiple devices of individual types with multiple gloves might give a better insight of this variation. A second limitation in this study was the low speed at which we activated the devices. A higher activation speed might slightly increase the activation forces and hysteresis because of viscous behavior.

\section{CONCLUSIONS}

We quantitatively tested nine voluntary opening prosthetic devices: four hooks and five hands. All hooks weighed less than a human hand. The masses of the gloved hands were similar to that of the human hand. All cable excursions were within the average movement range for shoulder activation. The hooks required a lower activation force and work than the hands. Nearly all hooks could pinch over $20 \mathrm{~N}$. The hands required a high activation force and could not pinch over $20 \mathrm{~N}$. Their use in daily life is expected to be limited. The Hosmer model 
5XA hook with three bands was the best tested hook. The Hosmer Sierra VO hand was the best tested hand.

Comparison with data of Corin et al. showed that VO prosthetic devices have not improved since 1987 [7]. Some newer devices even performed worse than the devices tested in 1987. The results of this study are helpful in selecting the right prosthetic device for a patient and in improving current devices. Future research should focus on reducing the mass of the cosmetic glove and hand mechanism, determining the comfortable activation force level for shoulder activation, decreasing the required activation force level, and increasing the pinch force of the hands.

\section{ACKNOWLEDGMENTS}

\section{Author Contributions:}

Study concept and design: D. H. Plettenburg, R. M. Bongers, G. Smit, C. K. Van der Sluis.

Acquisition of data: G. Smit.

Analysis and interpretation of data: G. Smit.

Drafting of manuscript: G. Smit, D. H. Plettenburg.

Critical revision of manuscript for important intellectual content:

R. M. Bongers, C. K. Van der Sluis.

Study supervision: D. H. Plettenburg, C. K. Van der Sluis.

Obtained funding: R. M. Bongers, D. H. Plettenburg, C. K. Van der Sluis.

Financial Contributions: The authors have declared that no competing interests exist.

Funding/Support: This material was based on work supported in part by OIM Stichting.

Additional Contributions: We would like to acknowledge Loth Fabenim B.V. for lending us the prosthetic hands and hooks. Also, we would like to acknowledge Otto Bock Benelux and Otto Bock Healthcare GmbH for lending us the Otto Bock hand and hook.

\section{REFERENCES}

1. Biddiss EA, Chau TT. Upper limb prosthesis use and abandonment: A survey of the last 25 years. Prosthet Orthot Int. 2007;31(3):236-57. [PMID:17979010] http://dx.doi.org/10.1080/03093640600994581

2. Shaperman J, Landsberger SE, Setoguchi Y. Early upper limb prosthesis fitting: When and what do we fit. J Prosthet Orthot. 2003;15(1):11-17. http://dx.doi.org/10.1097/00008526-200301000-00004

3. Shaperman J, LeBlanc M, Setoguchi Y, McNeal DR. Is body powered operation of upper limb prostheses feasible for young limb deficient children? Prosthet Orthot Int. 1995;19(3):165-75. [PMID:8927528]
4. Kejlaa GH. Consumer concerns and the functional value of prostheses to upper limb amputees. Prosthet Orthot Int. 1993; 17(3):157-63. [PMID:8134275]

5. LeBlanc MA. Innovation and improvement of body-powered arm prostheses: A first step. Clin Prosthet Orthot. 1985;9(1): 13-16.

6. Smit G, Plettenburg DH. Efficiency of voluntary closing hand and hook prostheses. Prosthet Orthot Int. 2010;34(4): 411-27. [PMID:20849359] http://dx.doi.org/10.3109/03093646.2010.486390

7. Corin JD, Holley TM, Hasler RA, Ashman RB. Mechanical comparison of terminal devices. Clin Prosthet Orthot. 1987;11(4):235-44.

8. Carlson LE, Long MP. Quantitative evaluation of bodypowered prostheses. Chicago (IL): American Society of Mechanical Engineers, Dynamic Systems and Control Division; 1988. p. 1-16.

9. LeBlanc M, Setoguchi Y, Shaperman J, Carlson L. Mechanical work efficiencies of body-powered prehensors for young children. J Assoc Child Prosthet Orthot Clin. 1992;27(3):70-75.

10. Keller AD, Taylor CL, Zahm V. Studies to determine the functional requirements for hand and arm prosthesis. Los Angeles (CA): Department of Engineering, University of California; 1947.

11. Van Der Niet O, Reinders-Messelink HA, Bongers RM, Bouwsema H, Van der Sluis CK. The i-LIMB hand and the DMC Plus hand compared: A case report. Prosthet Orthot Int. 2010;34(2):216-20. [PMID:20470060] http://dx.doi.org/10.3109/03093641003767207

12. Taylor CL. The biomechanics of the normal and of the amputated upper extremity. In: Klopsteg PE, Wilson PD, editors. Human limbs and their substitutes. New York (NY): McGraw-Hill; 1954. p. 169-221.

13. Chandler RF, Clauser CE, McConville JT, Reynolds HM, Young JW. Investigation of inertial properties of the human body. Dayton (OH): Wright-Patterson Air Force Base, Aerospace Medical Research Laboratory; 1975. Report No.: DOT HS-801 430.

14. Shaperman J, Setoguchi Y, LeBlanc M. Upper limb strength of young limb deficient children as a factor in using body powered terminal devices: A pilot study. J Assoc Child Prosthet Orthot Clin. 1992;27(3):89-96.

15. Monod H. Contractility of muscle during prolonged static and repetitive dynamic activity. Ergonomics. 1985;28(1): 81-89. [PMID:3996380] http://dx.doi.org/10.1080/00140138508963115

16. Van Lunteren A, Van Lunteren-Gerritsen GH. On the use of prostheses by children with a unilateral congenital forearm defect. J Rehabil Sci. 1989;2(1):10-12.

17. Biddiss E, Beaton D, Chau T. Consumer design priorities for upper limb prosthetics. Disabil Rehabil Assist Technol. 
JRRD, Volume 49, Number 4, 2012

2007;2(6):346-57. [PMID:19263565]

http://dx.doi.org/10.1080/17483100701714733

18. Jones LE, Davidson JH. Save that arm: A study of problems in the remaining arm of unilateral upper limb amputees. Prosthet Orthot Int. 1999;23(1):55-58. [PMID:10355644]

19. Herder JL, Cool JC, Plettenburg DH. Methods for reducing energy dissipation in cosmetic gloves. J Rehabil Res Dev. 1998;35(2):201-9. [PMID:9651892]

Submitted for publication July 15, 2011. Accepted in revised form November 1, 2011.

This article and any supplementary material should be cited as follows:
Smit G, Bongers RM, Van der Sluis CK, Plettenburg DH. Efficiency of voluntary opening hand and hook prosthetic devices: 24 years of development? J Rehabil Res Dev. 2012;49(4):523-34.

http://dx.doi.org/10.1682/JRRD.2011.07.0125

ResearcherID: Gerwin Smit, MSc: B-9994-2012; Raoul M. Bongers, MSc, PhD: C-1094-2012; Corry K. Van der Sluis, MD, PhD: C-1102-2012; Dick H. Plettenberg, MSc, PhD: C-1078-2012

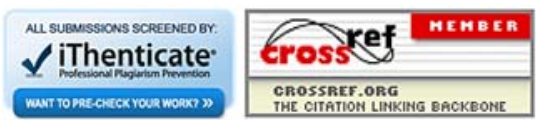

